\title{
Activité Anti-Hyperglycémiante Et Élucidation Structurale Des Composés Isolés Des Feuilles De Spondias Purpurea L. (Anacardiaceae)
}

\author{
Armel Diatta \\ Doctorant en Chimie Université Assane Seck de Ziguinchor/Sénégal \\ Anastasie Manga \\ Maitre Assistante, Biochimiste Université Assane Seck de \\ Ziguinchor/Sénégal \\ Abdoulaye Gassama \\ Maître de Conférences, Chimiste Université Assane Seck de \\ Ziguinchor/Sénégal \\ Catherine Lavaud \\ Professeur, Chimiste Université de Reims/France
}

Doi: 10.19044/esj.2017.v13n36p312 URL:http://dx.doi.org/10.19044/esj.2017.v13n36p312

\begin{abstract}
Spondias purpurea L. (Anacardiaceae) is a Senegalese traditional pharmacopoeia plant, which leaves are used in the treatment of various diseases. The leaves' ethanolic extract exhibited antihyperglycemic activity on models of temporary hyperglycemia. The tests were performed on normoglycemic rats with a glucose tolerance test. The phytochemical investigation of the leaves resulted in the isolation of four flavonoids (quercetin 9, kaempferol 10, quercetin-3- $\beta$-glucopyranoside 6, kaempferol3-ß-galactopyranoside 8), five phenolic compounds (2,4-dihydroxybenzoic acid 2, 4-hydroxybenzoic acid 5, protocatechic 4-ß-glucoside acid 1, hydroxycynnamic acid 7, 5-caffeoylquinic acid 3) and one tannin (Strictinin 4). The known isolated compounds were characterized by NMR and massspectrometry. The present study constitutes the first phytochemical examination of the leaves and the anti-hyperglycemic activity of the ethanolic extract of Spondias Purpurea L.
\end{abstract}

Keywords: Spondias purpurea L., antidiabetic, flavonoids, phenolic compounds, tannin 


\section{Résumé}

Spondias purpurea L. (Anacardiaceae) est une plante de la pharmacopée traditionnelle sénégalaise, dont les feuilles sont utilisées dans le traitement de diverses maladies. L'extrait éthalonique des feuilles a présenté une activité anti-hyperglycémiante sur des modèles d'hyperglycémie temporaire. Les tests ont été réalisés chez des rats normoglycémiques, sur un test de tolérance au glucose. L'étude phytochimique des feuilles a permis d'isoler quatre flavonoïdes (quercétine 9, kaempférol 10, quercétine-3- $\beta$-glucopyranoside 6, kaempférol-3- $\beta$-galactopyranoside 8), cinq composés phénoliques (acide 2,4-dihydroxybenzoïque 2, acide 4hydroxybenzoïque $\mathbf{5}$, acide protocatéchique 4- $\beta$-glucoside $\mathbf{1}$, acide hydroxycynnamique 7, acide 5-caffeoylquinique 3) et un tanin (Strictinine 4) connus. Les composés isolés connus ont été caractérisés par la RMN et la spectrométrie de masse. La présente étude constitue le premier examen phytochimique des feuilles et l'activité anti-hyperglycémiante de l'extrait éthanolique de Spondias Purpurea L.

Mots clés : Spondias purpurea L., antidiabétique, flavonoïdes, composés phénoliques, tanin

\section{Introduction}

Spondias purpurea L. appartient à la famille des Anacardiaceae dont de nombreuses espèces sont utilisées comme plantes médicinales (Wiart et al., 2006).

L'espèce Spondias purpurea L. s'est forgée une réputation considérable en médecine traditionnelle au Brésil, en République Dominicaine, en Haïti et dans plusieurs pays africains. Les feuilles, l'écorce et les racines de cette plante sont couramment utilisées au Sénégal et dans d'autres pays africains pour traiter diverses pathologies.

Au-delà de son importance économique (jus, gelées, glaces et boissons alcoolisées), l'étude ethno pharmacologique de cette espèce a montré qu'elle est utilisée en médecine populaire dans le traitement des maladies telles que la diarrhée, le diabète, le cholestérol (Nascimento et al., 2011; Dantas et al., 2012), l'inflammation et la gastrite (Franco et al., 2006).

En outre, des études phytochimiques des pelures de fruits de $S$. purpurea $\mathrm{L}$. ont permis d'isoler des acides phénoliques et des flavonoïdes $O$ glucosides, confirmant l'importance des fruits comme source d'antioxydants naturels (Engels et al., 2012). Nolasco (Nolasco et al., 2016) a réalisé un screening phytochimique de l'extrait éthanolique des feuilles de Spondias purpurea $L$., et a détecté la présence d'alcaloïdes, de tanins, de composés 
phénoliques, de quinones, de coumarines, de glucosides cardiotoniques et de triterpénoïdes.

Nous présentons dans cette étude pour la première fois l'isolement et l'élucidation structurale de quatre flavonoïdes, de cinq composés phénoliques et d'un tanin de l'extrait éthanolique des feuilles de Spondias purpurea L. (Anacardiaceae) et une évaluation pharmacologique de son activité anti-hyperglycémiante sur des modèles d'hyperglycémie temporaire.

\section{Matériel et Méthodes}

\section{Matériel végétal}

Les feuilles de Spondias purpurea L. (Anacardiaceae) ont été récoltées au mois de Janvier 2016 au quartier de Kandé (région de Ziguinchor, Sénégal). Elles ont été séchées pendant un mois et demi à l'abri des rayons solaires à la température ambiante $\left(27^{\circ} \mathrm{C}\right)$ au Laboratoire de Chimie et Physique des Matériaux (LCPM) de 1'Université Assane Seck de Ziguinchor (Sénégal). La plante a été authentifiée par le Professeur Emmanuel BASSENE, laboratoire de Pharmacognosie et Botanique de la Faculté de Médecine, de Pharmacie et d'Odontologie de l'Université Cheikh Anta Diop de Dakar (UCAD). Un spécimen a été déposé à l'herbier du dit laboratoire sous le numéro 2016/ 024.

\section{Extraction et fractionnement}

Les drogues séchées ont été broyées à l'aide d'un broyeur (type Bradender OHG Duisburg). La poudre fine (724 g) ainsi obtenue après pulvérisation a été utilisée comme matière première pour les extractions. Un épuisement successif de la poudre a été réalisé par des solvants de polarités croissantes (cyclohexane, éther, dichlorométhane, éthanol, eau). En effet, $724 \mathrm{~g}$ de poudre fine des feuilles de Spondias purpurea L. (Anacardiaceae) ont été introduits dans un ballon de $5 \mathrm{~L}$ contenant $3500 \mathrm{ml}$ de cyclohexane et le tout porté à ébullition pendant $4 \mathrm{~h}$. Le résidu obtenu après évaporation de l'extrait est mis dans un verre de montre et placé sous la hotte pour une évaporation complète du solvant d'extraction. Le marc issu de l'extrait cyclohexane est repris successivement dans les mêmes conditions que précédemment par $3500 \mathrm{ml}$ d'éther, de dichlorométhane, d'éthanol et d'eau. Les filtrats obtenus donnent respectivement après évaporation un résidu cyclohexanique, éthylique, dichlorométhanique, éthanolique et aqueux (Tableau I).

\section{Purification de la fraction éthanolique par HPLC semi-prépartive}

Une masse de $150 \mathrm{mg}$ du résidu sec de la fraction éthanolique a été purifiée à l'Université de Reims, Institut de Chimie Moléculaire de Reims (ICMR UMRS CNRS 6229), Groupe Isolement - Structure à l'aide d'un 
appareil HPLC semi-préparative de type 'Dionex',

'Solanum', Interchrom- UPtisphere Strategy ${ }^{\circledR}$ C18-2, $5 \mu \mathrm{m}, 100$ A, 250x10 $\mathrm{mm}$ avec un système de solvant $\mathrm{CH}_{3} \mathrm{CN} / \mathrm{H}_{2} \mathrm{O}$ avec un gradient $5-12 \%$ pendant $9 \mathrm{mn}$ puis 22-28,4\% pendant $12 \mathrm{mn}$ avec un débit de $4 \mathrm{ml} / \mathrm{mn}$. Les dix (10) produits purs (Tableau II) ont été collectés à l'aide d'un collecteur automatique.

Les spectres IR ont été enregistrés sur un spectromètre Nicolet Avatar 320 FT-IR. Les spectres UV/VIS ont été obtenus en utilisant un spectrophotomètre UV / VIS Philips PU 8720. Les spectres RMN $\left({ }^{1} \mathrm{H},{ }^{13} \mathrm{C}\right.$, COSY, HSQC, HMBC) ont été enregistrés sur un spectromètre Bruker Avance III sonde TCI DRX-600, fonctionnant à $600 \mathrm{MHz}$ pour ${ }^{1} \mathrm{H}$ et $150 \mathrm{MHz}$ pour ${ }^{13} \mathrm{C}$. Les constantes de couplage ont été exprimées en Hz. Les spectres de masse à haute résolution (HRESIMS) et ESIMS (mode à ions positifs) ont été enregistrés en utilisant le micro-instrument Micromass ESIQ-TOF (Manchester, Royaume-Uni).

\section{Matériel animal}

Des rats de souche wistar de poids moyen compris entre 127 et $170 \mathrm{~g}$ ont été utilisés. Ces rats ont été élevés à l'animalerie du Département de Chimie de l'Université Assane Seck de Ziguinchor (UASZ).

\section{Essais chez des rats normo-glycémiques}

Les rats ont été répartis en 2 lots de 5 puis mis à jeun pendant 14 heures avant l'expérience. Des prélèvements de sang ont été effectués sur les rats avant administration des produits. Les rats ont été ensuite gavés comme suit : le premier lot avec de l'eau physiologique (contrôle) à $10 \mathrm{mg} / \mathrm{kg}$, le deuxième lot avec l'extrait éthanolique de feuilles de Spondias purpurea L. à la dose de $300 \mathrm{mg} / \mathrm{kg}$. Des prélèvements de sang ont été effectués toutes les heures pendant 4 heures.

\section{Essais sur un test de tolérance au glucose}

Les rats ont été répartis en 2 lots de 5 puis mis à jeun pendant 14 heures avant l'expérience. Le premier prélèvement sanguin a été effectué 90 min avant gavage (T-90) pour déterminer la glycémie de base. Immédiatement après, les rats ont été gavés avec de l'eau physiologique (10 $\mathrm{mg} / \mathrm{kg}$ ), ou de l'extrait éthanolique de feuilles de Spondias purpurea L. (300 $\mathrm{mg} / \mathrm{kg}$ ). Un deuxième prélèvement sanguin a été effectué à T0, suivi de l'administration per os d'une solution de glucose $(4 \mathrm{~g} / \mathrm{kg})$. D'autres prélèvements de sang ont été effectués toutes les 30 min pendant $120 \mathrm{~min}$. 


\section{Dosage du glucose}

Le dosage a été réalisé à l'aide d'un glucomètre de type "système FreeStyle papillon vision'”.

\section{Analyse statistique}

Les résultats ont été exprimés sous la forme d'une moyenne \pm erreur standard à la moyenne. Ils ont été comparés à l'aide du test « $\mathrm{t} »$ de Student. La différence entre deux moyennes a été considérée comme significative lorsque $\mathrm{p}<0,05, \mathrm{n}=5$ est le nombre d'expériences dans chaque groupe.

\section{Résultats et discussion :}

Le but de cette étude était d'évaluer l'activité pharmacologique de l'extrait éthanol sur des rats normo-glycémiques, sur un modèle d'hyperglycémie temporaire puis d'élaborer une stratégie d'isolement et de détermination structurale des composés chimiques de cet extrait.

\section{Rendements de l'extraction}

Les résultats de l'extraction sont consignés dans le tableau I. Nous retenons dans ce tableau la richesse en composé polaire de la feuille de Spondias Purpurea L. En effet, Pereira (Pereira et al., 2015) a décrit une étude phytochimique d'une fraction enrichie en flavonoïdes des feuilles de $S$. venulosa, et le profil phénolique de cinq espèces représentatives de Spondias: S. dulcis, S. mombin, S. purpurea , S. venulosa et Spondias sp. Toutes les espèces analysées ont montré une composition phénolique similaire avec la présence majeure de flavonol O-glycosides, mais sans détection des aglycones respectifs, ce qui est cohérent avec l'information précédente pour les pelures de $S$. purpurea (Engels et al., 2012).

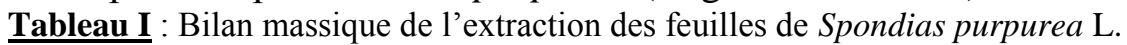

\begin{tabular}{ccc}
\hline Solvants & (Anacardiaceae). & \\
Cyclohexane & Masses $(\mathrm{g})$ & Rendement $(\%)$ \\
Ether & 19,90 & 2,74 \\
Dichlorométhane & 2,24 & 0,30 \\
Ethanol & 6,82 & 0,94 \\
Eau & 60 & 8,28 \\
& 76 & 10,49
\end{tabular}

\section{Essais chez des rats normo-glycémiques}

L'administration de l'eau physiologique, à la dose de $10 \mathrm{mg} / \mathrm{kg}$ per os, n'entraine pas une modification de la glycémie de base chez les rats normo-glycémiques au bout de $240 \mathrm{mn}$ d'observation $(0.74 \pm 0.02$ vs $0.65 \pm 0.04 \mathrm{~g} / \mathrm{l})(\mathrm{n}=5$ est le nombre d'expériences dans chaque groupe). Des résultats similaires ont été obtenus avec l'administration de l'extrait éthanol 
des feuilles à la dose de $300 \mathrm{mg} / \mathrm{kg}$ per os où on note une légère baisse de la glycémie entre T1 et T2 $(0.67 \pm 0.03$ vs $0.65 \pm 0.02 \mathrm{~g} / \mathrm{l})$.

Chez des rats normo-glycémiques, l'extrait éthanolique des feuilles de Spondias purpurea L. n'a pas d'effet significatif sur la glycémie de base (Figure 1).

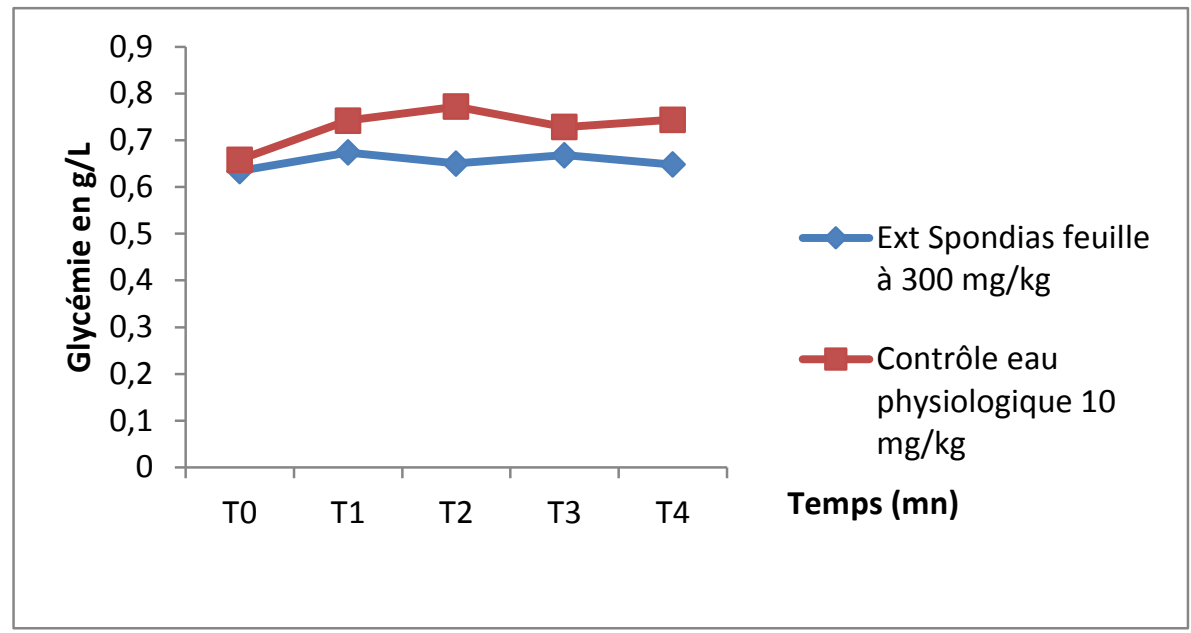

Figure 1: Variation de la glycémie chez des rats normo-glycémiques traités avec l'extrait éthanol des feuilles de Spondias purpurea L. (Anacardiaceae).

\section{Essais sur un test de tolérance au glucose}

Le prétraitement par l'extrait éthanol des feuilles de Spondias purpurea à la dose de $300 \mathrm{mg} / \mathrm{kg}$ per os a un effet préventif sur le pic hyperglycémique observé lors du test de tolérance au glucose. En effet, dans le groupe contrôle, la variation du pic d'hyperglycémie est de $1.38 \pm 0.02$ vs $0.82 \pm 0.03 \mathrm{~g} / \mathrm{l}$. Cette variation est moins importante dans le groupe ayant subi un prétraitement à l'extrait éthanol des feuilles de Spondias purpurea $(1.06 \pm 0.04$ vs $0.67 \pm 0.02 \mathrm{~g} / \mathrm{l})$ contrairement à celle du groupe témoin. Ce résultat pourrait s'expliquer par la présence de métabolites secondaires dans l'extrait éthanolique de Spondias purpurea. Le pic d'hyperglycémie est suivi par une chute rapide de la glycémie au bout de $30 \mathrm{mn}$ (T30 àT60) puis une évolution progressive aux valeurs de base au bout d'une heure. Cette diminution de l'hyperglycémie pourrait s'expliquer par un épuisement du taux de glucose reçu (Figure 2).

En effet, sur le plan phytochimique de notre étude, une molécule connue de la littérature, kaempférol $\mathbf{1 0}$ qui aurait des propriétés de baisser le taux de glucose sérique et du glucose dans le muscle soléaire du rat aussi efficacement que l'insuline (Behbahani et al., 2014; Vinayagam et al., 2015) a été isolée et caractérisée dans l'extrait éthanolique de feuilles de Spondias purpurea $\mathrm{L}$. 


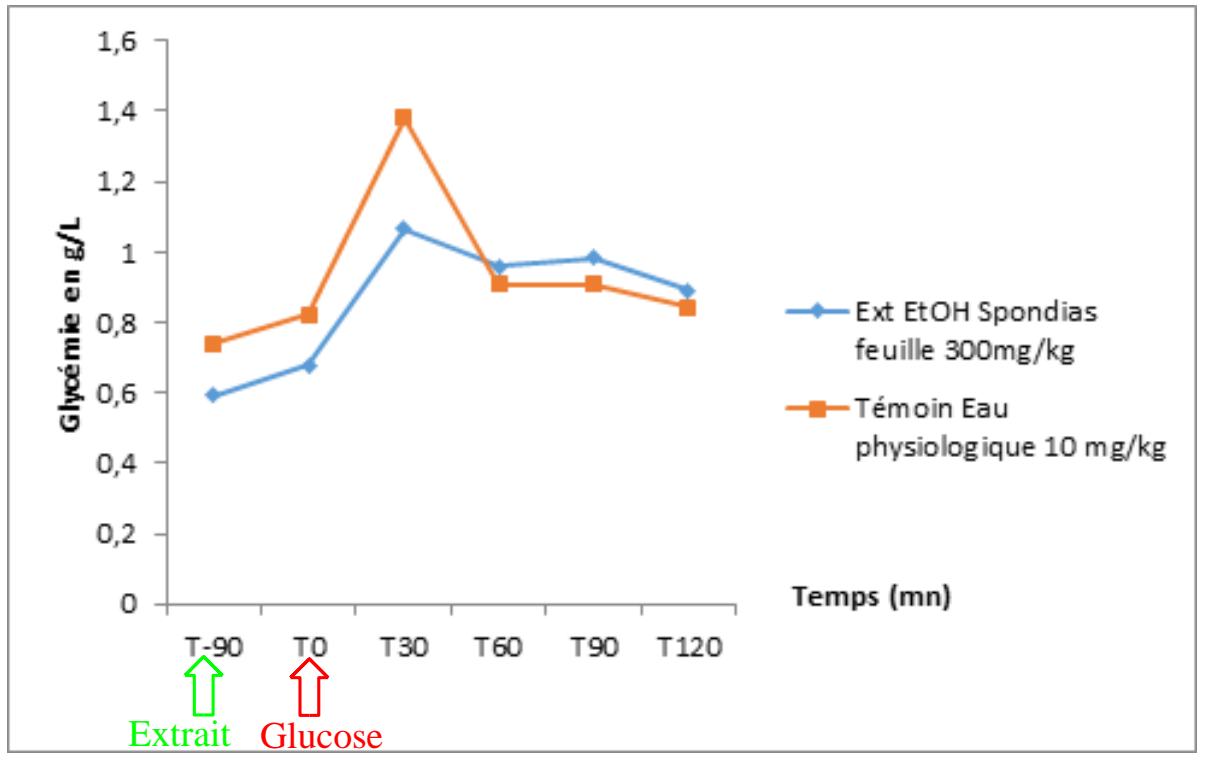

Figure 2: Variation de la glycémie chez des rats soumis à un test de tolérance de glucose après traitement avec l'extrait éthanol des feuilles de Spondias purpurea L. (Anacardiaceae).

Les résultats de l'isolement par HPLC semi-préparative en phase inverse de l'extrait éthanol des feuilles de Spondias purpurea L. sont consignés dans le tableau II. Les composés connus 1-10 ont été facilement identifiés par leurs données spectrales et par comparaison avec les composés correspondants rapportés dans la littérature comme étant quercétine 9, kaempferol 10, quercétine-3- $\beta$-glucopyranoside 6, kaempferol-3- $\beta$ galactopyranoside 8, acide 2,4-dihydroxy benzoïque 2, acide 4-hydroxy benzoïque 5, acide protocatéchique 4- $\beta$-glucoside $\mathbf{1}$, acide hydroxycynnamique 7, acide 5-caffeoylquinique 3 et Strictinine 4 (Figure 3) (Sambandam et al., 2016; Lim et al., 2007; Foo et al., 2000; Zhu et al., 2013; Abdullah et al.,tr 2016; Lu et al., 2016; Lee et al., 2016; Cho et al., 1998; Forino et al., 2015; Kraus, 2013).

Tableau 2 : Résultats de la purification par HPLC de l'extrait éthanolique des feuilles de Spondias purpurea $\mathrm{L}$.

$\begin{array}{cccc}\text { Composés } & \text { Temps de rétention }(\text { Tr en } \mathrm{mn}) & \mathrm{UV} \lambda_{\max }(\mathrm{nm}) & \text { Masses }(\mathrm{mg}) \\ \mathbf{1} & 6,195 & 193,1 ; 206,3 ; 286,1 & 4 \\ \mathbf{2} & 7,252 & 204,2 ; 215,8 ; 260,4 & 3,5 \\ \mathbf{3} & 9,306 & 196,4 ; 204,9 ; 207,1 & 4 \\ \mathbf{4} & 10,008 & 214,1 ; 216,1 ; 219,7 & 6 \\ \mathbf{5} & 11,254 & 192,7 ; 256,1 & 5 \\ \mathbf{6} & 12,502 & 196,1 ; 255,0 ; 367,7 & 6 \\ \mathbf{7} & 13,001 & - & 3,5 \\ \mathbf{8} & 13,375 & 193,0 ; 265,7 ; 348,1 & 4 \\ \mathbf{9} & 17,369 & 200,7 ; 368,2 ; 372,8 & 4,6\end{array}$




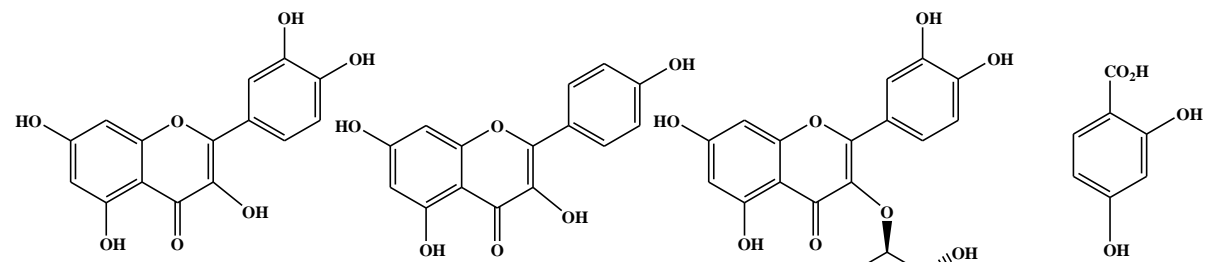<smiles>C/C=C/c1ccc([Al])cc1</smiles>

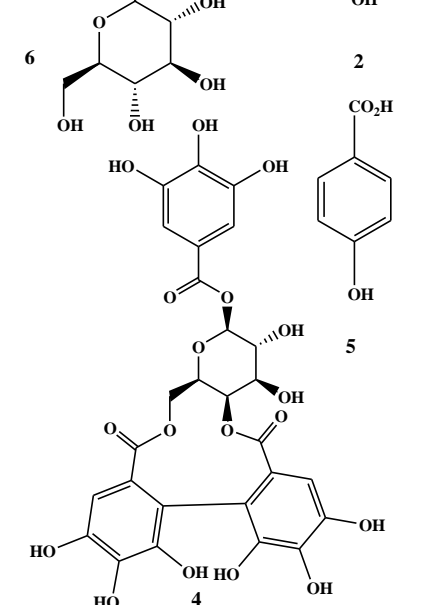

Figure 3 : structure des composés isolés : quercétine 9, kaempferol 10, quercétine-3- $\beta$ glucopyranoside 6, kaempferol-3- $\beta$-galactopyranoside $\mathbf{8}$, acide 2,4-dihydroxy benzoïque $\mathbf{2}$, acide 4-hydroxy benzoïque 5 , acide protocatéchique 4- $\beta$-glucoside $\mathbf{1}$, acide hydroxycynnamique 7, acide 5-caffeoylquinique 3 et Strictinine 4

L'élucidation de ces molécules dans les feuilles de Spondias purpurea L. confirme les études ethnobotaniques antérieures et les aspects thérapeutiques intéressants dans l'utilisation de cette plante. Les feuilles de Spondias purpurea L. (Anarcadiacea) sont une source importante de flavonoïdes, résultat non observé par Nolasco (Nalasco et al., 2016). La présence de flavonoïdes, qui constituent une classe importante de métabolites secondaires chez les Anacardiaceae, présente un aspect important des espèces de Spondias (Engels et al., 2012). En effet, ces composés jouent divers rôles biologiques dans la protection des plantes et leurs propriétés antioxydantes et chélatantes sont responsables de plusieurs avantages pour la santé humaine (Heim et al., 2002).

Des études antérieures ont montré que la quercétine 9 pourrait avoir des activités antioxydantes, antiinflammatoires, immunomodulatrices, anticancéreuses et gastro-protectrices (Li et $a l ., 2008$; Thuy et $a l .$, 2016). Le kaempférol 10 isolés de la plante médicinale Securigera securidaca aurait une activité anti-VIH-1 puissante, et des propriétés de baisser le taux de glucose sérique et du glucose dans le muscle soléaire du rat aussi 
efficacement que l'insuline (Behbahani et al., 2014; Vinayagam et al., 2015). La quercétine 3-O- $\beta$-D-glucopyranoside (isoquercitrine) 6 aurait une activité antivirale contre l'arbovirus MAYV (Santos et al., 2014). D’autres études ont montré que l'isoquercitrine possède d'excellentes activités antioxydantes mais n'exerce pas d'effets protecteurs au système biologique (Panat et al., 2015). Le kaempferol-3-O- $\beta$-D-galactoside 8, extrait de la partie aérienne de Calluna vulgaris L. aurait des activités anti-inflammatoires, antinociceptives, antioxydantes et antiprolifératives significatives contre le cancer (Orhan et al., 2007; Said et al., 2010). Des études menées sur l'acide 2,4-dihydroxy benzoïque (2) ont montré une forte inhibition de l' $\alpha$ glucosidase avec des $\mathrm{CI}_{50}$ intéressantes (Abdullah et al., 2016). L'acide 5caffeoylquinique (3), composé contenu dans le café vert a des potentialités hypoglycémiantes et hypolipidémies plus élevées que l'acide chlorogénique (CGA) (Forino et al., 2015; Farah et al., 2008; Liu et al., 2013).

$\mathrm{Au}$ plan phytochimique, l'isolement et la caractérisation de dix composés a montré que l'extrait éthanolique des feuilles de Spondias purpurea L. (Anacardiaceae) est riche en composés phénoliques et en flavonoïdes.

Plusieurs études ont décrit l'effet sur le glucose sanguin d'extraits de plantes riches en flavonoïdes. En effet, la fraction acétate d'éthyle d'E. alatus riche en flavonoïdes aurait des propriétés stimulantes sur la libération d'insuline (Fang et al., 2008). Il en n'est de même de l'effet antihyperglycémiant d'un flavonol dérivé de fleurs d'Hibiscus vitifolius Linn, sur un test de tolérance au glucose (Ragunathan et al., 1994).

L'effet anti-hyperglycémiant de l'extrait éthanol des feuilles de Spondias purpurea L. observé dans cette étude sur un test de tolérance au glucose, pourrait être lié à la présence de flavonoïdes.

\section{Conclusion}

Cet article rapporte pour la première fois une étude bioactive de l'activité anti- hyperglycémiante et phytochimique de l'extrait éthanolique des feuilles de Spondias purpurea L. L'extrait éthanolique des feuilles de Spondias purpurea L. a conduit à l'isolement d'un tanin, de quatre flavonoïdes et de cinq composés phénoliques. Un effet anti-hyperglycémiant de l'extrait éthanolique des feuilles de Spondias purpurea L. sur un test de tolérance au glucose a été démontré. Un essai sur le diabète de type 2 permettra de confirmer cette activité. Par conséquent, cette étude fournit une base moléculaire pour comprendre l'utilisation de cette plante dans la médecine traditionnelle sénégalaise. 


\section{Remerciements}

Les auteurs remercient l'Institut de Chimie Moléculaire (ICMR), Groupe d'Isolement et Structure (IS) de Reims, pour les caractérisations; le Gouvernement Sénégalais et la coopération Française pour l'attribution de la bourse à M. Armel Diatta.

\section{References:}

1. Abdullah, N. H., Salim, F., \& Ahmad, R. (2016). Chemical Constituents of Malaysian U. cordata var. ferruginea and Their in Vitro $\alpha$-Glucosidase Inhibitory Activities. Molecules, 21, p. 525; doi:10.3390/molecules21050525

2. Behbahani, M., Sayedipour, S., Pourazar, A., \& Shanehsazzadeh, M. (Décembre, 2014). In vitro anti-HIV-1 activities of kaempferol and kaempferol-7-Oglucoside isolated from Securigera securidaca. Research in Pharmaceutical Sciences, 9, pp. 463-469.

3. Cho, J.-Y., Moon, J.-H., Seong, K.-Y., \& Park, K.-H. (1998). Antimicrobial Activity of 4-Hydroxybenzoic Acid and trans 4 Hydroxycinnamic Acid Isolated and Identified from Rice Hull. Bioscience, Biotechnology, and Biochemistry, 62, pp. 2273-2276.

4. Dantas, A. M., \& Santos, V. L. (2012). Avaliação da Toxicidade e Atividade Antiulcerogênica das Folhas de Spondias purpurea L. [s.l.] Universidade Estadual da Paraíba, pp. 5-23.

5. Engels, C., Gräter, D., Esquivel, P., Jiménez, V. M., Gänzle M. G., \& Schieber, A. (2012). Characterization of phenolic compounds in Jacote (Spondias purpurea L.) peels by ultra-high-performance liquid chromatography/eletrospray ionization mass spectrometry. Food Research International, 46, pp. 557-562.

6. Fang, X. K., Gao, Y., Yang, H. Y., Lang, S. M., Wang, Q. J., Yu, B.Y., Zhu, D. N. (2008) Alleviating effects of active fraction of Euonyus alatus abundant in flavonoids on diabetic mice. American Journal of Chinese Medicine, 36, pp.125-140.

7. Farah, A., Monteiro, M., Donangelo, C. M., \& Lafay, S. (2008). Chlorogenic Acids from Green Coffee Extract are Highly Bioavailable in Humans. Biochemical, Molecular, and Genetic Mechanisms, pp.2309-2315.

8. Foo, L.Y., Lu, Y., Molan, A. L., Woodfield, D.R., \& McNabb, W. C. (2000). The phenols and prodelphinidins of white clover flowers. Phytochemistry, 54, pp. 539-548.

9. Forino, M., Tenore, G. C., Tartaglione, L., Carmela, D. A., Novellino, E., $\&$ Ciminiello, P. (2015). (1 $S, 3 R, 4 S, 5 R)$ 5-O-Caffeoylquinic acid: 
Isolation, stereo-structure characterization and biological activity. Food Chemistry, 178, pp. 306-310.

10. Franco, E. A. P., \& Barros, R.F.M. (2006). Uso e diversidade de plantas medicinais no quilombo Olho d'agua dos Pires, Esperantina, Piaui. Revista Brasileira de Plantas Medicinais, 8, pp. 78-88.

11. Heim, K. E.; Tagliaferro, A. R.; Bobilya, D. J.; (2002), Flavonoid antioxidants: chemistry, metabolism and structure-activity relationships, Journal Nutritional Biochemistry, 13, pp. 572-584.

12. Iwai, K., Kishimoto, N., Kakino, Y., Mochida, K., \& Fujita, T. (2004). In Vitro Antioxidative Effects and Tyrosinase Inhibitory Activities of Seven Hydroxycinnamoyl Derivatives in Green Coffee Beans. Journal of Agricultural and Food Chemistry, 52, pp.4893-4898.

13. Kraus, G. A., \& Maury, W. (2013). Tannin Inhibitors of HIV. Patent Application Publication US 2013/0252909 A1

14. Lee, S. R., Clardy, J., Senger, D. R., Cao, S., \& Kim, K. H. (2016). Iridoid and phenylethanoid glycosides from the aerial part of Barleria lupulina. Revista Brasileira de Farmacognosia. Brazilian journal of pharmacology, http://dx.doi.org/10.1016/j.bjp.2016.01.002

15. Li, M., \& Xu, Z. (2008). Quercetin in a Lotus Leaves Extract May be Responsible for Antibacterial Activity. Archives of Pharmacal Resaerch, 31, pp. 640644.

16. Lim, Y.-H., Kim I.-H., et Seo, J.-J. (2007). In vitro Activity of Kaempferol Isolated from the Impatiens balsamina alone and in Combination with Erythromycin or Clindamycin against Propionibacterium acnes. The Journal of Microbiology, 45, pp. 473477.

17. Liu, H., Zhang, X., Wu, C., Wu, H., Guo, P., Xu, X. (2013). Antihyperlipidemic caffeoylquinic acids from the fruits of Pandanus tectorius Soland. Journal of Applied Pharmaceutical Science, 3, pp. 016-019.

18. Lu, Q., Sun, Y., Shu, Y., Tan, S., Yin, L., Guo, Y., \& Tang, L. (2016). HSCCC Separation of the Two Iridoid Glycosides and Three Phenolic Compounds from Veronica ciliata and Their in Vitro Antioxidant and Anti-Hepatocarcinoma Activities. Molecules, 21, p. 1234, doi: $\underline{10.3390 / \text { molécules21091234 }}$

19. Nascimento, J. M., \& Conceicao, G. M. (2011). Plantas medicinais e indicaçoes terapêuticas da comunidade quilombola olho d'agua do Raposo, Caxias, Maranhao, Brasil. Revista de Biologia e Farmàcia, 6, pp. 138-151. 
20. Nolasco, N. L. (IC), dos Santos, M. M. O (IC), Costa, R. de O. (IC), Santos, A. P. (IC), de Rezende, L. C. (PQ), de Sousa, \& V. R. (PQ) (2016). Análise fitoquímica dos galhos de Spondias purpurea L. (siriguela). Sociedade Brasileira de Química (SBQ), 39 a Reunião Anual da Sociedade Brasileira de Química: Criar e Empre ender, p. 1.

21. Orhan, I., Küpeli, E., Terzioglu, S., \& Yesilada, E. (2007). Bioassayguided isolation of kaempferol-3-O - $\beta$-D-galactoside with antiinflammatory and antinociceptive activity from the aerial part of Calluna vulgaris L. Journal of Ethnopharmacology, 114, pp. 32-37.

22. Panat, N. A., Amrute, B. K., Bhattu, S., Haram, S. K., Sharma, G. K., \& Ghaskadbi, S. S. (2015). Antioxidant profiing of C3 quercetin glycosides: Quercitrin, Quercetin 3- $\beta$-D-glucoside and Quercetin 3O-(6"-O-malonyl)- $\beta$-Dglucoside in cell free environment. Free Radicals and Antioxidants 5, pp. 90-100.

23. Pereira, C., de Oliveira, L. L.; Goncalves, R., Amaral, A. C. F., Kuster, R. M., \& Sakuragui, C. M. (2015). Phytochemical and phylogenetic analysis of Spondias (Anacardiaceae), Quimica Nova, 38, PP. 813-816.

24. Ragunathan, V., Sulochana, N. (1994). A new flavonol bioside from the flower of Hibiscus vitifolius Linn. and its hypoglycaemic activity. Journal of the Indian Chemical Society, 71 ; 705-706.

25. Said, A., Tundis, R., Hawas, U. W., El-Kousy, S. M., Rashed, K., Menichini, F., Bonesi, M., Huefner, A., Loizzo, M. R., \& Menichini, F. (2010). In vitro Antioxidant and Antiproliferative Activities of Flavonoids from Ailanthus excelsa (Roxb.) (Simaroubaceae) Leaves. Zeitschrift für Naturforschung C, 65, pp.180 - 186.

26. Sambandam, B., Thiyagarajan, D., Ayyaswamy A., \& Raman, P. (2016). Extraction and isolation of flavonoid quercetin from the leaves of Trigonella foenum-graecum and their anti-oxidant activity. International Journal of Pharmacy and Pharmaceutical Sciences, 8, pp. 120-124.

27. Santos, A. E., Kuster, R. M., Yamamoto, K. A., Salles, T. S., Campos R., Meneses, M. DF., Soares, M. R., \& Ferreira, D. (2014). Quercetin and quercetin 3- O-glycosides from Bauhinia longifolia (Bong.) Steud. Show anti-Mayaro virus activity. Parasites \& Vectors, 7, p. 130.

28. Thuy, B. T. P., Nhung, N. T. A., Duong, T., Trung, P. V., Quang, N. M., Dung, H.T. K., \& Tat, P. V. (2016). Prediction of anticancer activities of cynaroside and quercetin in leaf of plants Cynara scolymus L and Artocarpus incisa L using structure-activity 
relationship. Cogent Chemistry, 2: 1212452, http://dx.doi.org/10.1080/23312009.2016.1212452.

29. Vinayagam, R., \& Xu, B. (2015). Antidiabetic properties of dietary flavonoids: a cellular mechanism review. Nutrition \& Metabolism, v. 02, (p. 60).

30. Wiart, C. (2006). Medicinal plants classified in the family Anacardiaceae. Medicinal Plants of Asia and the Pacific, p. 191. Boca Raton, FL: CRC Press.

31. Zhu, Y., Liu Y., Zhan, Y., Liu, L., Xu, Y., Xu, T., \& Liu, T. (2013). Preparative Isolation and Purification of Five Flavonoid Glycosides and One Benzophenone Galloyl Glycoside from Psidium guajava by High-Speed Counter-Current Chromatography (HSCCC). Molecules, 18, pp.15648-15661. 